\title{
Periostin in the Cancer Stroma of Mycosis Fungoides Palmaris et Plantaris: A Case Report and Immunohistochemical Study
}

\author{
Kayo Tanita Taku Fujimura Aya Kakizaki Sadanori Furudate \\ Masato Mizuashi Akiko Watabe Setsuya Aiba \\ Department of Dermatology, Tohoku University Graduate School of Medicine, \\ Sendai, Japan
}

\section{Key Words}

Periostin - Mycosis fungoides palmaris et plantaris - Tumor-associated macrophages - M2 polarization · Prognosis

\begin{abstract}
Mycosis fungoides palmaris et plantaris (MFPP) is a rare variant of mycosis fungoides limited to the palms and soles. Although little is known about the pathogenesis of MFPP, this variant of mycosis fungoides presents a relatively good prognosis. In this report, we describe an 85year-old Japanese man with MFPP. Immunohistochemical staining revealed the dense deposition of periostin in the cancer stroma, as well as infiltration of $\mathrm{CD} 163^{+} \mathrm{CD}_{206}{ }^{-}$tumorassociated macrophages (TAMs), which suggested the phenotypes of TAMs were not polarized to the M2 phenotype in the lesional skin of MFPP. Our present case might suggest one of the possible reasons for the good prognosis of MFPP.

(C) 2016 The Author(s)

Published by S. Karger AG, Basel
\end{abstract}

\section{Introduction}

Mycosis fungoides palmaris et plantaris (MFPP) is an uncommon variant of mycosis fungoides (MF) limited to the palms and soles, first described by Resnik et al. in 1995 [1]. Since only 10 English papers about MFPP have been previously published [2], little is known about its pathogenesis. In this report, we describe a case of MFPP showing a dense deposition of periostin (POSTN) in the cancer stroma, which might modulate the tumor microenvi-

\section{KARGER}

Taku Fujimura

Department of Dermatology

Tohoku University Graduate School of Medicine

Seiryo-machi 1-1, Aoba-ku, Sendai 980-8574 (Japan)

E-Mail tfujimura1@mac.com 
Case Reports in
Dermatology
Case Rep Dermatol 2016;8:31-35

\begin{tabular}{l|l}
\hline DOI: $10.1159 / 000444162$ & (c) 2016 The Author(s). Published by S. Karger AG, Basel \\
\end{tabular}

www.karger.com/cde

Tanita et al.: Periostin in the Cancer Stroma of Mycosis Fungoides Palmaris et Plantaris: A Case Report and Immunohistochemical Study

ronment through tumor-associated macrophages (TAMs) [3]. Our present case might suggest one of the possible reasons for the good prognosis of MFPP.

\section{Case Report}

An 85-year-old Japanese man visited our outpatient clinic with a 2-year history of pruritic erythema on his scalp. His condition had been treated in a private clinic as pustulosis palmaris et plantaris with topical steroid and maxacalcitol, but they were ineffective. On his initial visit, physical examination revealed erosive, atrophic, well-demarcated scaly erythema on his right palm and right sole (fig. 1a, b). A biopsy specimen showed atypical large lymphocytes densely infiltrated mainly in the upper dermis with involvement of the overlying epidermis (fig. 2a). The epidermotropic lymphocytes formed Pautrier's microabscesses. Immunohistochemical (IHC) staining revealed that these atypical lymphocytes, which were distributed from the upper layer of the stratum spinosum to the dermis, were positive for CD3, CD4 and CD5, and negative for CD7 and CD8. A full blood count and biochemical profile revealed eosinophilia (19.7\%), and a significantly high level of serum IL-2 receptor (925 U $/ \mathrm{ml}$ ). We screened for possible metastatic lesions with positron emission tomography and computed tomography but found no evidence of metastasis. From the above findings, we diagnosed this patient as having MFPP. We administered radiation therapy 40 Gy in 20 fractions to the right palm and sole, and the erosive, atrophic erythema then disappeared.

To further investigate the possible immunological background of the MFPP, we employed IHC staining for POSTN, CD163 and CD206. IHC staining revealed the dense deposition of POSTN within the superficial to deep dermis (fig. 2b), where CD163+ macrophages were densely infiltrated (fig. 2c). Few $\mathrm{CD}_{206}{ }^{+}$cells were detected in the lesional skin of the dermis (fig. 2d).

\section{Discussion}

MFPP is a rare variant of MF limited to the palms and soles [2, 4, 5]. Since only a small number of cases have been reported in English, the pathogenesis and biological behaviors of MFPP are still incompletely understood [2]. Among the published reports, Nakai et al. [2] reviewed 24 cases of MFPP, which suggested that patients with MFPP present a relatively good prognosis, and that radiotherapy is one of the best therapies for MPFF [2]. Excimer laser therapy (308 $\mathrm{nm}$ ) and bexarotene are also effective [4,5] and, to our knowledge, no patients have died from disease progression [2].

POSTN is an extracellular matrix protein that modulates the production of a series of chemokines from macrophages and fibroblasts $[3,6]$. As we previously reported, the deposition of POSTN is prominent in early stage (patch to plaque stage) of conventional MF [3]. In addition, previous reports also suggested that in vitro stimulation of monocyte-derived macrophages by POSTN induces the production of chemokines such as CXCL5 and CXCL10 [3], which correlates with the tumor-formation of MF in the early stage [7]. In another report, Zhou et al. [8] reported that POSTN secreted from glioblastoma stem cells increases the M2 TAMs in the tumor site, resulting in the growth of glioblastoma multiformes. These reports suggest that the POSTN/TAM axis might contribute to the progression of the early stage of MF.

In our present case, unlike conventional MF in the tumor stage [3], the dense infiltration of atypical lymphoid cells as well as dense deposition of POSTN were observed throughout 
Tanita et al.: Periostin in the Cancer Stroma of Mycosis Fungoides Palmaris et Plantaris: A Case Report and Immunohistochemical Study

the dermis. In addition, the main population of TAMs was CD163+CD206- macrophages, which suggested that TAMs were not polarized to M2 macrophages in MFPP. As a previous reports suggested, M2 macrophages produce CCL17 and CCL22 [9-11], which recruit regulatory T cells (Tregs) in the tumor microenvironment [10]. Since a large population of Tregs is associated with a poor prognosis in various types of human cancer [12], and the depletion of M2-like TAMs delays the cutaneous T-cell lymphoma development in vivo [7], the inhibition of M2 polarization in TAMs might be connected with the progression of MF. Concerning our present case, the phenotypes of TAMs were not polarized to the M2 phenotype, which might be responsible for the good prognosis in MFPP. Since this report presented a single case of MFPP, and since we did not directly prove the relationships between immunomodulatory effects of POSTN and prognosis of MFPP, further analysis of the mechanisms underlying this phenomenon may provide fundamental insights into the mechanisms of POSTN with MFPP. Such clarifications will need to be addressed in future investigations.

\section{Statement of Ethics}

The patient gave written informed consent.

\section{Disclosure Statement}

The authors declare no conflicts of interest.

\section{References}

1 Resnik KS, Kantor GR, Lessin SR, Kadin ME, Chooback L, Cooper HS, Vonderheid EC: Mycosis fungoides palmaris et plantaris. Arch Dermatol 1995;131:1052-1056.

-2 Nakai N, Hagura A, Yamazato S, Katoh N: Mycosis fungoides palmaris et plantaris successfully treated with radiotherapy: case report and mini-review of the published work. J Dermatol 2014;41:63-67.

-3 Furudate S, Fujimura T, Kakizaki A, Kambayashi Y, Asano M, Watabe A, Aiba S: The possible interaction between periostin expressed by cancer stroma and tumor-associated macrophages in developing mycosis fungoides. Exp Dermatol 2016;25:107-112.

4 Jin SP, Jeon YK, Cho KH, Chung JH: Excimer laser therapy (308 nm) for mycosis fungoides palmaris et plantaris: a skin-directed and anatomically feasible treatment. Br J Dermatol 2010;163:651-653.

5 Miernik B, Schmidt V, Technau-Hafsi K,Kern JS, Meiss F: Alitretinoin in the treatment of palmoplantar mycosis fungoides: a new and promising therapeutic approach. Clin Exp Dermatol 2015;40:445-447.

-6 Uchida M, Shiraishi H, Ohta S, Arima K, Taniguchi K, Suzuki S, Okamoto M, Ahlfeld SK, Ohshima K, Kato S, Toda S, Sagara H, Aizawa H, Hoshino T, Conway SJ, Hayashi S, Izuhara K. Periostin, a matricellular protein, plays a role in the induction of chemokines in pulmonary fibrosis. Am J Respir Cell Mol Biol 2012;46:677686.

7 Wu X, Schulte BC, Zhou Y, Haribhai D, Mackinnon AC, Plaza JA, Williams CB, Hwang ST: Depletion of M2-like tumor-associated macrophages delays cutaneous T-cell lymphoma development in vivo. J Invest Dermatol 2014;134:2814-2822.

-8 Zhou W, Ke SQ, Huang Z, Flavahan W, Fang X, Paul J, Wu L, Sloan AE, McLendon RE, Li X, Rich JN, Bao S: Periostin secreted by glioblastoma stem cells recruits M2 tumour-associated macrophages and promotes malignant growth. Nat Cell Biol 2015;17:170-182.

-9 Martinez FO, Gordon S, Locati M, Mantovani A: Transcriptional profiling of the human monocyte-tomacrophage differentiation and polarization: new molecules and patterns of gene expression. J Immunol 2006;177:7303-7311.

10 Fujimura T, Kambayashi Y, Furudate S, Asano M, Kakizaki A, Aiba S: Receptor activator of nuclear factor kappa-B ligand (RANKL) promotes the production of CCL17 from RANK+ M2 macrophages. J Invest Dermatol 2015;135:2884-2887. 


\section{Case Reports in Dermatology}

\begin{tabular}{l|l}
\hline Case Rep Dermatol 2016;8:31-35 \\
\hline DOI: $10.1159 / 000444162$ & $\begin{array}{l}\text { (c) 2016 The Author(s). Published by S. Karger AG, Basel } \\
\text { www.karger.com/cde }\end{array}$ \\
\hline
\end{tabular}

Tanita et al.: Periostin in the Cancer Stroma of Mycosis Fungoides Palmaris et Plantaris: A Case Report and Immunohistochemical Study

11 Fujimura T, Kambayashi K, Furudate S, Kakizaki A, Aiba S: Immunomodulatory effect of bisphosphonate risedronate sodium on $\mathrm{CD} 163^{+}$Arginase $1^{+} \mathrm{M} 2$ macrophages: the development of a possible supportive therapy for angiosarcoma. Clin Dev Immunol 2013;2013:325412.

12 Nishikawa H, Sakaguchi S: Regulatory T cells in cancer immunotherapy. Curr Opin Immunol 2014;27:1-7.

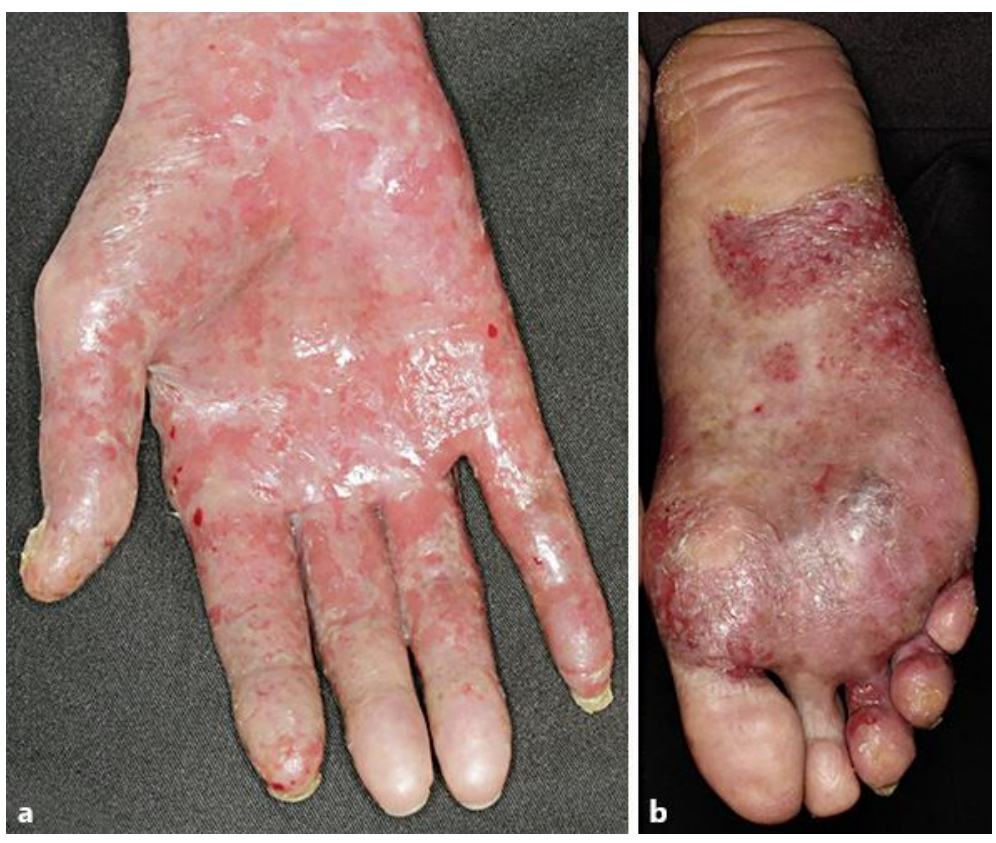

Fig. 1. Erosive, atrophic, well-demarcated scaly erythema with on the patient's right palm (a) and right sole (b). 
Tanita et al.: Periostin in the Cancer Stroma of Mycosis Fungoides Palmaris et Plantaris: A Case Report and Immunohistochemical Study
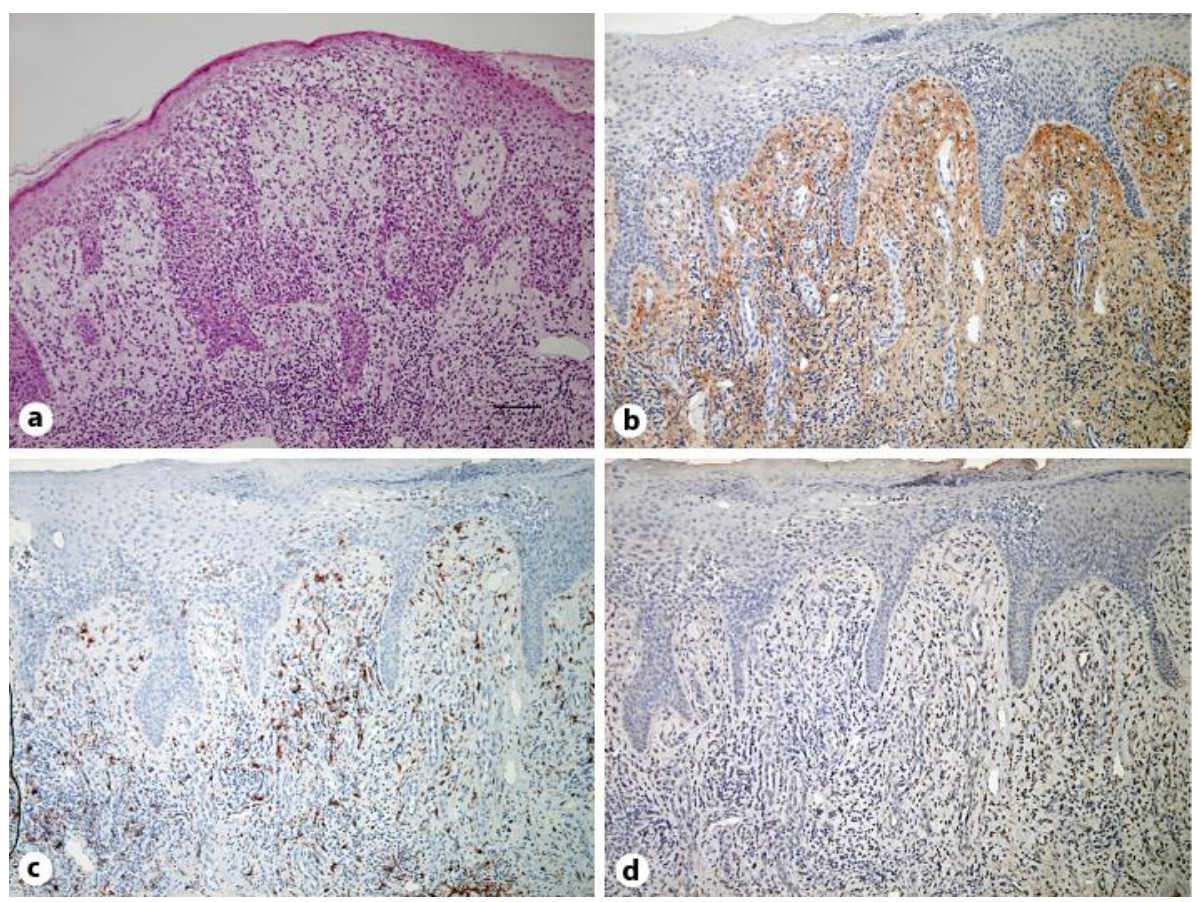

Fig. 2. Atypical large lymphocytes densely infiltrated mainly in the upper dermis with involvement of the overlying epidermis (a). Paraffin-embedded tissue samples were deparaffinized and stained with antiPOSTN (b), anti-CD163 (c) and anti-CD206 antibodies (d). The sections were developed with liquid permanent red. Original magnification: $\mathbf{a} \times 200, \mathbf{b}-\mathbf{d} \times 100$. 